\title{
DIABETES MELLITUS: RELATO DE EXPERIÊNCIA SOBRE PROJETO DE EXTENSÃO DURANTE A PANDEMIA DO COVID-19
}

\section{Anna Virgínia Bisognin Felice ${ }^{1}$; Elisangela Colpo ${ }^{2}$; Lilian Oliveira de Oliveira ${ }^{3}$; Minéia Weber Blattes ${ }^{4}$}

\section{RESUMO}

É através da educação em saúde que os profissionais da área da saúde exercem seu papel de educadores, capacitando a população para atuar de maneira qualificada no cuidado e autocuidado. $O$ objetivo deste trabalho foi descrever as atividades desenvolvidas em um projeto de extensão sobre diabetes mellitus. Tratase de um relato de experiência da bolsista do referido projeto desenvolvido no período de agosto de 2020 a julho de 2021. Esta vivência proporcionou um aumento nos conhecimentos sobre o tema proposto, capacitando os participantes do projeto a socializarem seus conhecimentos científicos adquiridos na universidade com a população em geral.

Palavras-chave: Distúrbio metabólico; educação em saúde; SARS-CoV-2.

Eixo Temático: Atenção Integral e Promoção à Saúde.

\section{INTRODUÇÃO}

Em dezembro de 2019, na cidade de Wuhan, na China, iniciou-se um surto de uma nova doença, denominada COVID-19, causada pelo coronavírus 2, que causa uma síndrome respiratória aguda grave (SARS-CoV-2), a qual a Organização Mundial da Saúde (OMS), em março de 2020, declarou ser uma pandemia.

Este cenário evidenciou que a tríade saúde-doença-cuidado pode ser influenciada pelas ações de educação em saúde, sobretudo para grupos de risco para o COVID-19, como os pacientes com diabetes mellitus (DM).

\footnotetext{
${ }^{1}$ Acadêmica do Curso de Farmácia. Bolsista PROBEX - Universidade Franciscana/UFN anna.felice@ufn.edu.br

${ }_{2}^{2}$ Professora do Curso de Nutrição - Universidade Franciscana/UFN - elicolpo@ufn.edu.br

${ }^{3}$ Professora do Curso de Fisioterapia - Universidade Franciscana/UFN - lilian.oliveira@ufn.edu.br

${ }^{4}$ Orientadora. Professora do Curso de Biomedicina - Universidade Franciscana/UFN -

mweber@ufn.edu.br
} 
O DM é em um distúrbio metabólico caracterizado por hiperglicemia persistente, decorrente de deficiência na produção de insulina ou na sua ação, ou em ambos os mecanismos (IDF, 2017). A hiperglicemia crônica pode comprometer a imunidade inata e a imunidade humoral. Ademais, o DM se associa com um estado inflamatório crônico de baixo grau que afeta a regulação da glicose e a sensibilidade periférica à insulina (IACOBELLIS, 2020).

Em geral, as pessoas com DM têm maior risco de desenvolver complicações quando se contaminam com a COVID-19 (YANG et al., 2020). Esta relação entre DM e COVID-19 também se evidenciou em outras epidemias causadas por outros coronavírus, como o causador do SARS em 2002 e da síndrome respiratória aguda do Oriente Médio (MERS) em 2012 (MEMISH et al., 2020).

A extensão universitária desenvolvida pelas universidades tem como ponto central o estabelecimento de vínculo entre a sociedade e as instituições de nível superior. Contudo, o cenário pandêmico instalado em 2020 forçou a necessidade de reformulação das atividades acadêmicas, entre elas, a condução dos projetos e atividades extensionistas (ARRUDA, 2020).

Frente ao exposto, este relato tem por objetivo descrever as atividades remotas de estudo e educação em saúde, desenvolvidas durante a pandemia da COVID-19, por um grupo multiprofissional de estudante e professores sobre a temática do diabetes mellitus.

\section{METODOLOGIA}

Este trabalho constitui um relato de experiência, vivenciado pela autora, bolsista de projeto de extensão na Universidade Franciscana. Trata-se de um estudo qualitativo, por métodos observacionais, o qual busca apresentar uma reflexão em âmbito profissional e de interesse da comunidade científica, estabelecendo conexões entre a teoria e a prática. A temática principal deste trabalho foi descrever as atividades remotas de estudo e educação em saúde para pacientes com DM, realizadas no período de agosto de 2020 a julho de 2021, organizadas por docentes e discentes da Universidade Franciscana e vinculados ao Grupo Multidisciplinar de 
EDUCAÇÃO, SAÚDE

ETECNOLOGIA

26 A 28 DE OUTUBRO DE 2021

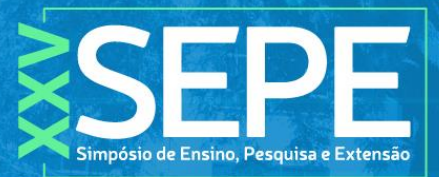

TRABALHO COMPLETO

Educação, Pesquisa e Extensão em Diabetes Mellitus da Universidade Franciscana - GEPED/UFN.

\section{RESULTADOS E DISCUSSÕES}

O DM é um grupo de doenças metabólicas caracterizadas por hiperglicemia resultante de defeitos na secreção de insulina, ação da insulina ou ambos. A hiperglicemia crônica do DM está associada a danos a longo prazo, disfunção e falência de diferentes órgãos, especialmente olhos, rins, nervos, coração e vasos sanguíneos (ADA, 2013).

A grande maioria dos casos de DM se enquadra em duas grandes categorias, o DM tipo 1, o qual a causa é uma deficiência absoluta de secreção de insulina. Os indivíduos com risco aumentado de desenvolver este tipo de DM podem frequentemente ser identificados por evidências sorológicas de um processo patológico autoimune que ocorre nas ilhotas pancreáticas e por marcadores genéticos. Na outra categoria, muito mais prevalente, DM tipo 2, a causa é uma combinação de resistência à ação da insulina e uma resposta inadequada de secreção de insulina compensatória. Na última categoria, um grau de hiperglicemia suficiente para causar alterações patológicas e funcionais em vários tecidos-alvo, mas sem sintomas clínicos, pode estar presente por um longo período de tempo antes que o DM seja detectado (SBD, 2019).

Em pacientes com DM infectados com o SARS-CoV-2 evidenciou-se um aumento nos níveis de interleucina- 6 e proteína $C$ reativa portanto, o estado próinflamatório próprio do DM pode favorecer a tempestades de citocinas e a resposta inflamatória sistêmica que acompanha a SARS em pacientes com COVID-19 (GUO et al., 2020).

Uma gama de estudos disponíveis mostrou que o DM é uma comorbidade que está associada a casos graves de SARS e ao aumento da mortalidade em pacientes que contraem o novo coronavírus e que apresentam variabilidade glicêmica (GUAN et al., 2020; YANG et al., 2020; HONG et al., 2014).

Com base nesta temática, e na sua importância durante a pandemia da COVID-19, o projeto de extensão foi desenvolvido por um grupo multiprofissional de 
professores e alunos da Universidade Franciscana, denominado Grupo Multidisciplinar de Educação, Pesquisa e Extensão em Diabetes Mellitus da Universidade Franciscana - GEPED/UFN.

Após a divulgação do projeto, formou-se um grupo com 16 integrantes de diversos cursos da área da saúde: biomedicina, enfermagem, farmácia, fisioterapia, medicina, nutrição e odontologia, orientados por professores da instituição.

O objetivo do projeto foi de realizar educação em saúde através do grupo de estudos no qual os alunos puderam estudar, entender e aprofundar os conhecimentos acerca do DM e sobre a importância do cuidado multiprofissional nesta doença. Ao longo do projeto, foi possível adquirir conhecimento sobre a doença, medicações, prevenção e tratamento nutricional, assim como as complicações que a doença pode causar nos pacientes.

Neste projeto, foi possível o aprofundamento os conhecimentos para poder socializar com a população em geral o quão importante é saber mais sobre a doença, prevenção e autocuidado, ressignificando sobre os cuidados e autocuidado em pacientes com a doença.

No decorrer dos primeiros meses foram realizados estudos sobre os diversos tipos de DM, sua fisiopatologia e complicações, para que todos os integrantes soubessem diferenciar os tipos da doença e entendessem a doença como um todo, e não somente dentro da sua área de atuação, proporcionando assim o entendimento do cuidado multiprofissional e facilitando o trabalho futuro em equipe.

O início do projeto se deu em meio a pandemia da COVID-19, na qual os pacientes com DM são grupos de risco, sobretudo os que apresentam variabilidade glicêmica. Assim, o grupo iniciou as atividades de disseminação dos conhecimentos através da rede social Instagram, utilizando uma linguagem acessível e de fácil compreensão pela comunidade em geral. Posto isso, cabe destacar que um baixo grau de escolaridade e uma bagagem de informações escassas sobre o assunto é fator de menor engajamento dos pacientes com diabetes no seu autocuidado e dificulta o manejo da doença (ALVARADO-MAREL et al., 2019).

No Instagram @gepedufn (figura 1) foram veiculados alguns materiais com intuito de transmitir conhecimento científico e de qualidade para a população 
EDUCAÇ̃O, SAÚDE

ETECNOLOGIA

26 A 28 DE OUTUBRO DE 2021

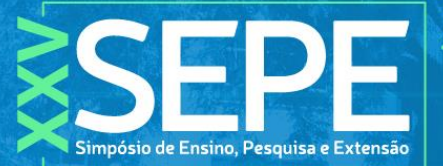

acadêmica e geral. É importante ressaltar que apesar de muitos grupos populacionais conviverem com o DM durante anos, poucos têm conhecimento básico sobre o assunto (BORBA AKOT, et al., 2015).
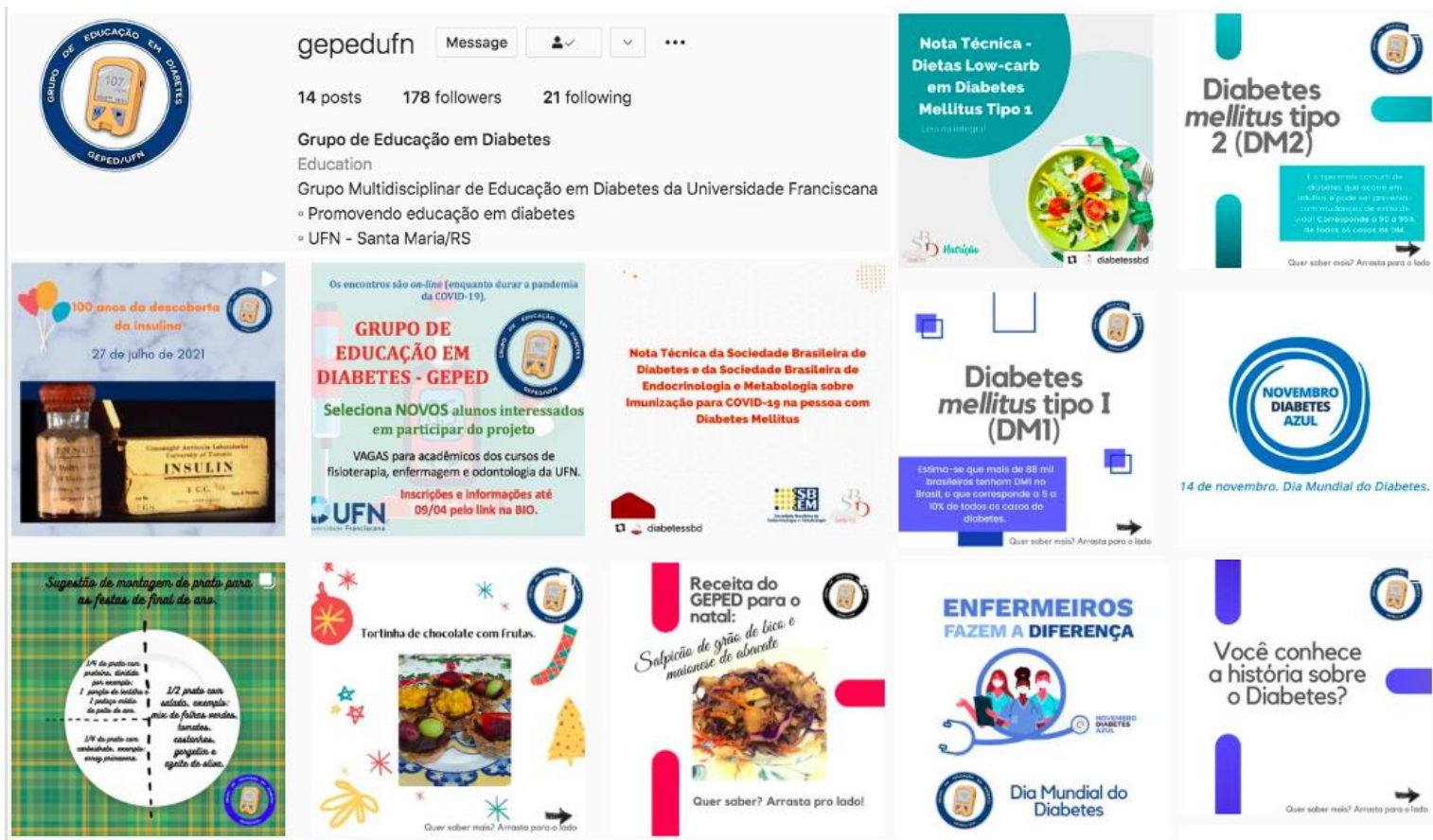

Figura 1: imagem do Instagram @gepedufn onde foram veiculados alguns materiais de educação em saúde.

Diante da nova realidade da pandemia, a forma de contato entre profissionais da área da saúde e pacientes teve que ser adaptada e sofreu algumas alterações promissoras para o enfrentamento desse novo momento (MARQUES et al., 2020). Assim, neste contexto, adaptamos as atividades para a realidade da pandemia da COVID-19, estreitando a relação entre os estudantes e a comunidade em geral, para as quais as publicações foram destinadas.

\section{CONCLUSÃO}

Os processos de educação em saúde são caracterizados pela disseminação de conhecimentos acerca de uma temática, sendo neste caso o DM. Objetivou-se com este projeto disseminar os conhecimentos científicos em saúde e promover mudança do estilo de vida desses pacientes, através de materiais produzidos em linguagem acessível para a população em geral e comunidade acadêmica. Além 
disso, os estudantes puderam aprofundar seus conhecimentos acerca da temática abordada no projeto, entendo a fisiopatologia da doença, suas complicações diretas e indiretas, assim como, entendendo as maneiras de evitá-las através do aumento da adesão de um estilo de visa saudável, bem como da importância de todos os profissionais da saúde no cuidado do paciente com diabetes.

Acredita-se que a proposta do grupo de estudo multiprofissional foi significativa, pois foi possível adquirir conhecimentos sobre o DM, ressignificando o conhecimento teórico e prático dos estudantes e auxiliando a sociedade no enfrentamento da doença e no cuidado do paciente com DM. A pandemia da COVID-19 impossibilitou o contato presencial com os pacientes, atividades as quais estão previstas para este momento de retorno gradual à presencialidade.

\section{AGRADECIMENTOS}

À Universidade Franciscana pelo apoio financeiro através da bolsa de projeto de extensão - PROBEX/UFN.

\section{REFERÊNCIAS}

ALVARADO-MARTEL D, et al. Identification of psychological factors associated with adherence to self-care behaviors amongst patients with type 1 diabetes. Journal Diabetes Research, v. 14, p. 1-10, 2019.

AMERICAN DIABETES ASSOCIATION. Diagnosis and Classification of Diabetes Mellitus. Diabetes Care, v. 36, n. 1, p. 67-74, 2013.

ARRUDA, E. P. Educação remota emergencial: elementos para políticas públicas na educação brasileira em tempos de Covid-19. Revista de Educação a Distância em Rede, v. 7, n. 1, p. 257-275, 2020.

BORBA AKOT, et al. Conhecimento sobre o diabetes e atitude para o autocuidado de idosos na atenção primária à saúde. Ciência \& Saúde Coletiva, v. 24, n. 1, p. 125-136, 2019. 
GUAN W., et al. Clinical Characteristics of Coronavirus Disease 2019 in China. N Engl J Med, v. 382, p. 1708-1720, 2020.

HONG K. W., et al. Clinical courses and outcomes of hospitalized adult patients with seasonal influenza in Korea, 2011-2012: Hospital-based Influenza Morbidity \& Mortality (HIMM) surveillance. J Infect Chemother, v. 20, p. 9-14, 2014.

INTERNATIONAL DIABETES FEDERATION. IDF Atlas. 8. ed. Bruxelas: International Diabetes Federation, 2017.

MARQUES L.C., et al. Covid-19: cuidados de enfermagem para segurança no atendimento de serviço pré- hospitalar móvel. Texto \& Contexto Enfermagem, v. 29 p. $1-12,2020$.

MEMISH Z.A., et al. Middle East respiratory syndrome. Lancet, v. 395, p. 1063-1077, 2020

SOCIEDADE BRASILEIRA DE DIABETES. Diretrizes Sociedade Brasileira de Diabetes 2019-2020. São Paulo: Editora Clannad, 2019.

Yang X., et al. Clinical course and outcomes of critically ill patients with SARS-CoV-2 pneumonia in Wuhan. China: A single-centered, retrospective, observational study. Lancet Respir Med., v. 8, p. 475-481, 2020. 